\title{
Non-elective thoracic surgery in patients supported by veno- venous (VV) or veno-arterial (VA) extracorporeal membrane oxygenation (ECMO)
}

\author{
Michael Ried ${ }^{1}$, Laura Sommerauer ${ }^{1}$, Till Markowiak ${ }^{1}$, Sigrid Wiesner ${ }^{1}$, Thomas Mueller ${ }^{2}$, Alois Philipp ${ }^{3}$, \\ Christoph Unterbuchner ${ }^{4}$, Marcus Creutzenberg ${ }^{4}$, Dirk Lunz ${ }^{4}$, Hans-Stefan Hofmann ${ }^{1}$ \\ ${ }^{1}$ Department of Thoracic Surgery, ${ }^{2}$ Department of Internal Medicine II, ${ }^{3}$ Department of Cardiothoracic Surgery, ${ }^{4}$ Department of Anaesthesiology, \\ University Medical Center Regensburg, Regensburg, Germany \\ Contributions: (I) Conception and design: M Ried, HS Hofmann; (II) Administrative support: A Philipp, T Mueller; (III) Provision of study materials \\ or patients: A Philipp, T Mueller, L Sommerauer; (IV) Collection and assembly of data: L Sommerauer, S Wiesner, A Philipp, T Mueller; (V) Data \\ analysis and interpretation: M Ried, L Sommerauer, T Mueller, S Wiesner; (VI) Manuscript writing: All authors; (VII) Final approval of manuscript: \\ All authors. \\ Correspondence to: Michael Ried, MD. Department of Thoracic Surgery, University Medical Center Regensburg, Franz-Josef-Strauß-Allee 11, 93053 \\ Regensburg, Germany. Email: michael.ried@ukr.de.
}

\begin{abstract}
The application of veno-arterial (VA) or veno-venous (VV) extracorporeal membrane oxygenation (ECMO) is still expanding worldwide in order to treat patients with severe cardiac and/ or respiratory failure. Pulmonary or pleural infections and severe thoracic trauma as some of the leading causes for acute lung failure necessitating VV ECMO might indicate urgent thoracic surgery with the aim to control bleeding, infection and to avoid septic multiorgan failure. Although miniaturized extracorporeal devices are used more common as a temporary support in these patients, concomitant bleeding complications and anticoagulation disorders are well known clinically relevant problems during ECMO support. Thoracic bleeding complications are frequently reported bleeding complications and often require surgical interventions. Non-elective thoracic surgery in patients with VA or VV ECMO warrants strict indications, because these patients have an extensively high perioperative risk profile and often suffer from postoperative complications with the need of surgical revision in approximately $60 \%$ of cases. Therefore, ECMO support should be performed whenever possible in specialised centers with an all-time available thoracic surgeon who has surgical experience in these jeopardised patients. The indication and optimal time for thoracic surgery as well as the perioperative management (coagulation management, ventilation, weaning, re-operations) should always be determined in an interdisciplinary setting.
\end{abstract}

Keywords: Extracorporeal membrane oxygenation (ECMO); thoracic surgery; thoracic bleeding; lung failure

Received: 19 August 2020; Accepted: 12 September 2020.

doi: $10.21037 /$ ccts-20-141

View this article at: http://dx.doi.org/10.21037/ccts-20-141

\section{Background}

The implantation of veno-arterial (VA) extracorporeal membrane oxygenation (ECMO) is mainly indicated in patients with acute cardiac failure after cardiac infarction, malignant arrhythmias or after cardiac surgery when other conservative resuscitation measures have failed (1). Venovenous (VV) ECMO is applied in patients with respiratory failure and acute respiratory distress syndrome (ARDS) due to infectious disease, severe trauma, after surgery or other reasons for damaged lung parenchyma $(2,3)$. In the summary of the Extracorporeal Life Support Organization (ELSO) Registry in 2019, a survival rate of $71 \%$ after extracorporeal life support (ECLS) and of $55 \%$ to hospital discharge or transfer was reported (4). 


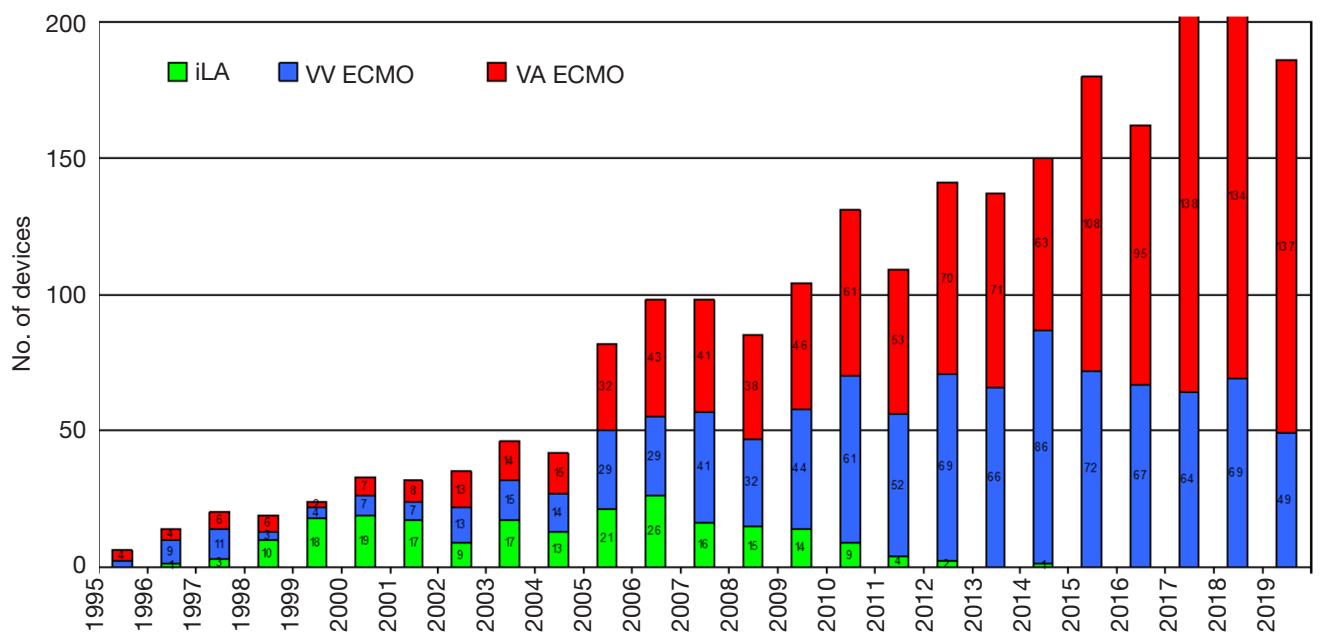

Figure 1 Overview of all extracorporeal devices implanted at the ECMO-center Regensburg. VV, veno-venous; VA, veno-arterial; ECMO, extracorporeal membrane oxygenation.

However, clinically relevant complications are manifold and should always be expected in the clinical setting during intensive care. Hemorrhage and thrombosis have been identified as major causes of morbidity and mortality in ECMO patients (5-8). Specifically, bleeding complications with often thoracic origin remain a clinically significant issue and raise the mortality rate $(9,10)$. Thoracic bleeding complications mostly occur spontaneous or after interventions including thoracic surgery. However, even after primarily successful surgical therapy and stopping of systemic anticoagulation, almost $50 \%$ of patients with intrathoracic bleeding are scheduled for repeated surgery due to recurrent bleeding (10).

Furthermore, infectious pleural effusion (e.g., pleural empyema) or other destroying pulmonary diseases (e.g., lung abscess, incarnating pneumonia) while on ECMO support sometimes necessitate invasive thoracic interventions (11-13).

In the following sections we describe the perioperative management in patients with VV ECMO therapy who also required non-elective thoracic surgery at our center. We give a short summary of thoracic bleeding complications and thoracic surgical procedures during ECMO support based on data of our ECMO working group. Furthermore, we want to give a tight review of the available literature.

\section{Regensburg ECMO-Registry}

The ECMO program at the University Medical Center
Regensburg, Germany, started first in 1995 and has steadily increased in the number of patients treated with extracorporeal support devices (Figure 1). Until the year 2001, patients with lung failure were predominantly treated with pumpless interventional lung assist (iLA ${ }^{\circledR}$ Xenios, Heilbronn, Germany). Later on, pump-driven devices (ECMO) were used more often and represent now a well-established technique in patients with acute cardiac and/or pulmonary failure. All patients are documented in the Regensburg ECMO-Registry and data were analysed for various studies. In the year 2014, our ECMO center became for the first time certified by the ELSO. In 2017, our ECMO-center was assigned with the platinum certificate of the ELSO.

\section{ECMO}

Miniaturized extracorporeal devices are used by standard as a temporary support in patients with cardiac failure or respiratory insufficiency after all conventional treatment options have failed (14). The selection of vessels (location, arterial or venous) for cannulation depends on the indication and anatomical situation. Percutaneous cannulation via Seldinger's technique is the preferred cannulation technique and has demonstrated a low complication rate. In our center, mainly three different ECMO systems are used, which are all coated with heparin, heparin-like substances or phosphorylcholine (15). The extracorporeal device consists of a membrane oxygenator, tubing and a centrifugal pump. Detailed technical and implantation data 
have been described by our working group in previous publications (16-18).

\section{Thoracic bleeding complications}

While patients with severe respiratory insufficiency were treated with VV ECMO in our center, we recognized clinically relevant bleeding complications, which is also described in the available literature $(19,20)$. Therefore, we performed a retrospective analysis of data from the Regensburg ECMO-Registry between December 2010 and December 2016. This study included all patients $(n=418)$ with severe respiratory failure supported with VV ECMO (10). Thoracic bleeding complications were the most frequent bleeding complication within the subgroup of patients with bleeding complications of any origin $(\mathrm{n}=97 ; 23.2 \%)$ and were documented in $9.6 \%$ of all patients $(n=40)$. These data were in line with those in the current literature and the ELSO-Registry of $2016(6,21)$. The thoracic bleedings were diagnosed approximately 10 days after ECMO implantation. It appeared in most patients without any intervention (spontaneously $40 \%$ ) due to coagulation disorders, but also after surgery (37.5\%), after interventions (20\%, e.g., chest tube placement) and late after trauma (2.5\%). These patients required significantly more blood products and coagulation factors. In all patients with persistent or recurrent bleeding, ECMO treatment was continued without systemic anticoagulation, as already reported (18).

A total of 24 patients $(60 \%)$ underwent urgent thoracic surgery and in nearly half of the patients (45.8\%) repeated surgery had to be performed due to recurrent postoperative bleeding complications. This high rate of re-operations was also shown in other studies $(11,22)$. Since some patients $(n=9 ; 37.5 \%)$ required thoracic surgery more than two times, all in all 69 thoracic surgical procedures were performed. Thoracotomy with open evacuation of a hemothorax, was performed 52 times (75.4\%), which was followed by primary surgery of pulmonary or pleural infections $(n=10)$ and other indications $(n=7)$. The residual patients $(n=16)$ were treated conservatively $(n=6)$ or interventionally $(n=10$; chest tube, interventional bronchoscopy). Patients with thoracic bleeding complications were significantly longer on ECMO and had a significantly higher mortality rate (52.5\%) compared to patients without bleeding complications (34.7\%). The in-hospital mortality after non-elective thoracic surgery due to bleeding was $41.7 \%$.

\section{Non-elective thoracic surgery}

In another study we analysed all consecutive patients with VV ECMO with the objective to evaluate the indications for and the outcome after non-elective thoracic surgery (13). Overall, in $6.9 \%(n=29)$ of patients non-elective thoracic surgery was performed, most frequently therapeutic due to hemothorax $(\mathrm{n}=13 ; 44.8 \%)$, followed by carnifying pneumonia/pulmonary abscess $(\mathrm{n}=5 ; 17.2 \%)$, pleural empyema $(\mathrm{n}=3 ; 10.3 \%)$ and others $(\mathrm{n}=3 ; 27.6 \%$; Figure 2$)$. In case of an unknown interstitial lung disease $(\mathrm{n}=5 ; 17.2 \%)$ diagnostic lung biopsy was performed. Primary thoracotomy was done in nearly all patients ( $\mathrm{n}=27 ; 93.1 \%)$, whereas only two patients $(6.9 \%)$ received video-assisted thoracoscopy. Initial thoracic surgery was performed mean 13.6 days after implantation of the ECMO. At least one re-operation was performed in half of the patients $(\mathrm{n}=15 ; 51.7 \%)$ and one third $(31.0 \%)$ had more than two operations. This resulted in a total of 70 thoracic surgical interventions (primary surgery $n=29$; repeated surgery due to complications $n=41$ ), which means approximately 2.4 operations per patient.

As mentioned above, a clinically relevant hemothorax presented the main indication for urgent surgery (71.4\%). Of these patients, $94 \%$ had previous thoracic interventions. In addition, postoperative thoracic bleeding after primary thoracic surgery was requisite in $90.2 \%$ (37/41) of operations (Figure 3). Patients who required thoracic surgery stayed significantly longer in hospital (57.3 vs. 32.8 days; $\mathrm{P}=0.031)$. The in-hospital mortality of patients after non-elective thoracic surgery was $44.8 \%$ and tended to be higher than in patients without thoracic surgery $(35.7 \%$; $\mathrm{P}=0.326)$.

\section{Anaesthesiological management}

If patients are scheduled for non-elective thoracic surgery while on ECMO, a preoperative evaluation together with the surgeon has to be performed. This includes the assessment and documentation of the ECMO settings and blood flow, risk of coagulopathy, platelet dysfunction and disseminated intravascular coagulation (DIC), type and cross for red blood cells, allocation of fresh frozen plasma (FFP) (if needed as well as checking for ECMO specific complications such as arrhythmias, anemia, bleeding, pneumothorax and thrombosis (23). Any required modification of ECMO support, changes in the cannulation strategy (right internal jugular dual lumen cannula versus femoral-jugular versus femoral-femoral) and vessel accesses 


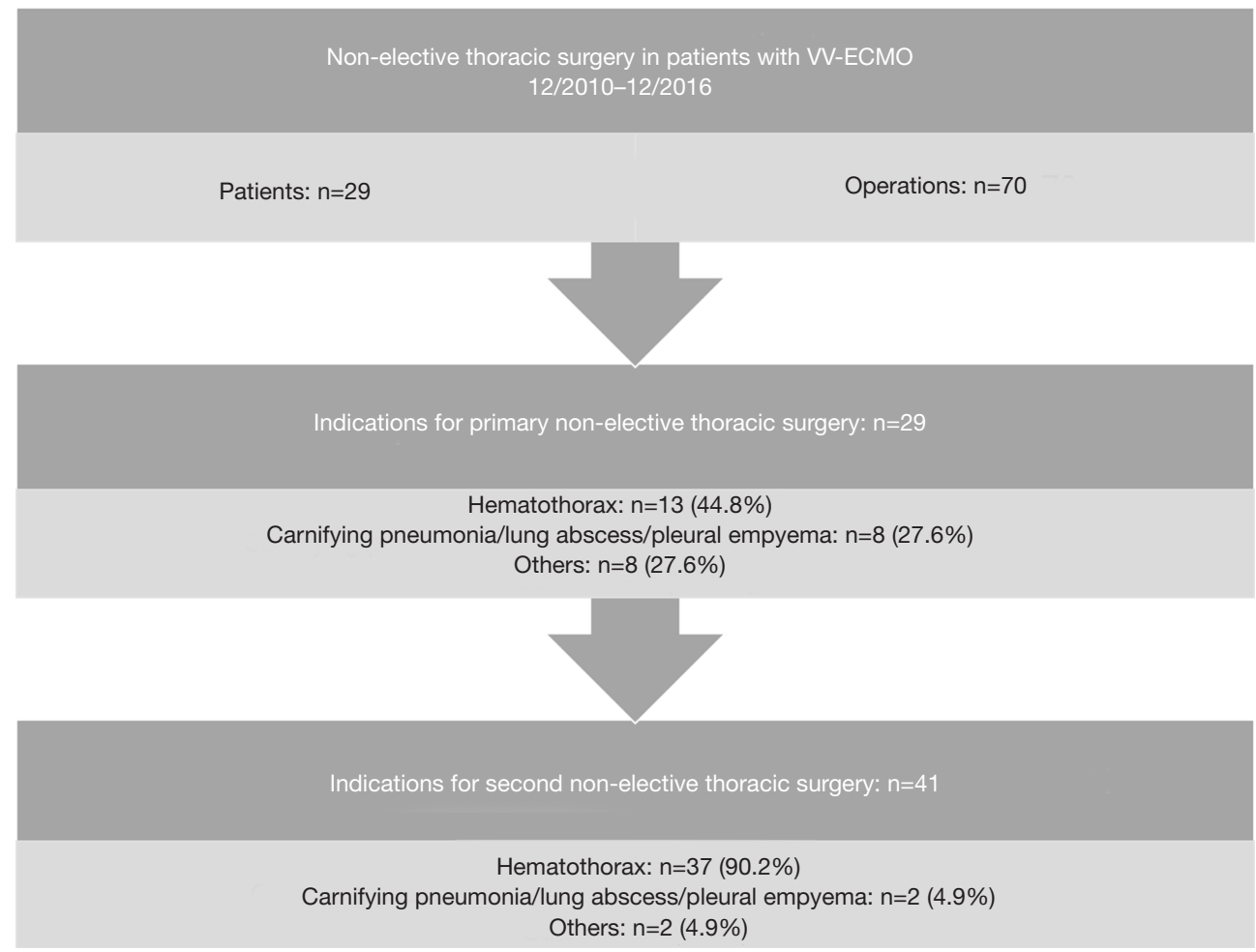

Figure 2 Indications for non-elective thoracic surgery in ECMO patients (10). VV, veno-venous; ECMO, extracorporeal membrane oxygenation.

should be discussed preoperatively and adapted to the planned procedure (24). Systemic anticoagulation with heparin should be stopped 4-6 hours before surgery if feasible and indicated. Required coagulation products as well as blood products should be ordered in time.

For transportation to the operating room and during the operation a practitioner dedicated to ECMO and capable of troubleshooting should always be available. Patients require little or no additional anaesthetic depending on baseline degree of sedation. Total intravenous anaesthesia (TIVA) is preferred because it allows for continuous delivery of anaesthetic especially if single-lung ventilation is needed. During VV ECMO the arterial line can be placed at any site. We use an anaesthetic depth monitoring to minimize the side effects of anaesthetics.

Transesophageal echocardiography is used to measure cardiac output, identify myocardial dysfunction as well as identify ECMO specific complications such as cannula malposition, presence of thrombus and hypovolaemia that can cause "chattering" of the ECMO lines $(25,26)$.

During surgery we routinely administer tranexamic acid intravenously (10 mg/kg bolus, $2 \mathrm{mg} / \mathrm{kg} / \mathrm{h}$ continuously) for antifibrinolytic effects in order to decrease the risk of diffuse bleedings especially from the pleural surface. Furthermore, patients undergoing non-elective major thoracic surgery with relevant blood loss may develop a deficiency of platelets, fibrinogen and other clotting factors, which will prolong coagulation even in the absence of heparin. Desmopressin is commonly used in the management of bleeding in ECMO patients, because a spectrum of acquired von Willebrand factor deficiency is common in ECMO patients (27). We use traditional coagulation labs and thromboelastometry to direct management of bleeding, this includes balancing the risk of thrombosis in the ECMO circuit against the patient and procedure specific risk of perioperative bleeding. Any surgical intervention increases the bleeding risk despite using ECMO without heparin (24). But withholding heparin and correction of coagulation abnormalities may increase the risk of postoperative thrombosis in the ECMO circuit or the oxygenator (28).

Our ventilation strategy consists of "ultra-protective ventilation", which maintains $\mathrm{V}_{\mathrm{T}}$ (tidal volume) of less than $4 \mathrm{~mL} / \mathrm{kg}$ predicted body weight and $\mathrm{P}_{\text {plat }}$ of less than 25 


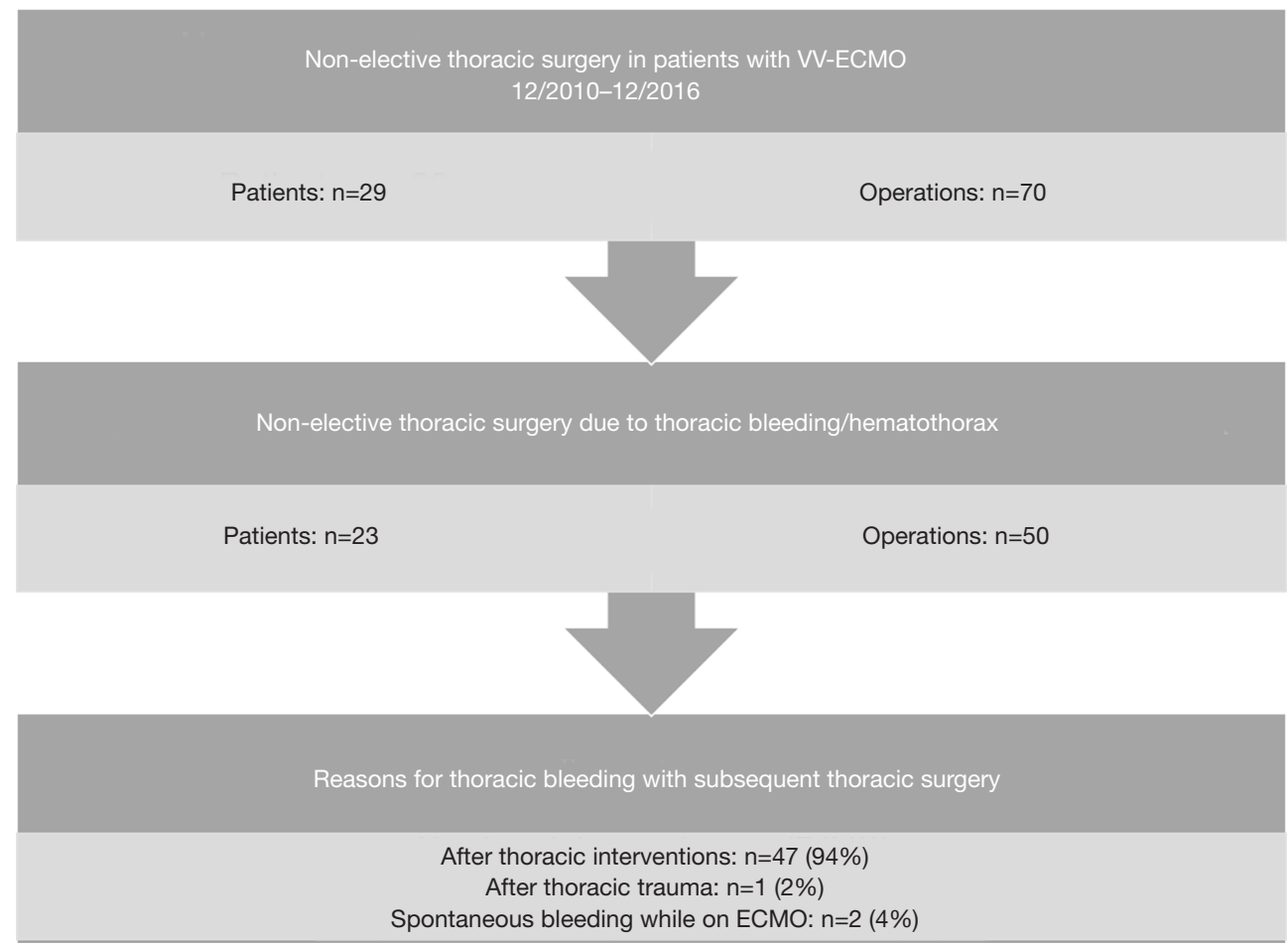

Figure 3 Non-elective thoracic surgery due to thoracic bleeding complications in patients with VV ECMO (13). VV, veno-venous; ECMO, extracorporeal membrane oxygenation.

$\mathrm{cmH}_{2} \mathrm{O}$, to reduce ventilation induced lung injury (VILI) $(29,30)$. The pump flow and sweep gas flow of the ECMO is adapted according to the results of frequent blood gas analysis, intraoperatively at least every 30 minutes. In case of hypoxemia we use the following strategies to improve oxygen delivery and oxygenation: blood transfusion, muscle relaxation, sedation, cooling, increasing ventilator $\mathrm{F}_{\mathrm{i}} \mathrm{O}_{2}$ (inspiratory oxygen fraction), increasing ventilator positive end-expiratory pressure (PEEP), increasing cardiac output or increasing oxygen flow to membrane lung (31).

\section{Perioperative intensive care management}

Anticoagulation on ECMO in the perioperative surgical setting is historically performed via unfractioned heparin (UFH), mainly based on considerations of experience and reversibility. However, there is still an ongoing debate on what might be the ideal anticoagulant drug and which goals [e.g., activated partial thromboplastin time (aPTT) $v s$. anti-activated factor $\mathrm{X}$ (anti-Xa) vs. activated clotting time (ACT)] should be achieved (32). In this context not only the risk of bleeding and thrombosis has to be balanced, but also patient specific and at the same time dynamic aspects like drug metabolism and organ dysfunction must be taken into account. Point of care coagulation monitoring devices like ROTEM ${ }^{\circledR}$ or TEG ${ }^{\circledR}$ might offer a more detailed insight into the patient's actual coagulation system but their superiority over global tests is not proven yet. By using UFH a continuous infusion with approximately $400-1,500$ IE/hour is started to achieve an aPTT of $45-55$ seconds. At this point once again, it is essential to emphasize that any anticoagulation strategy constantly has to be reevaluated and adapted to the patient's individual needs. In case of bleeding complications, which perioperatively may be caused by low platelet count, platelet dysfunction, hypofibrinogenemia, acquired von Willebrand syndrome and possibly FXIII deficiency, intravenous heparin should be paused. In addition, any form of pharmacological procoagulative intervention (e.g., coagulation or blood components, antifibrinolytics)—even the administration of rFVIIa - can be administered on ECMO (33). As bleeding remains the most common complication on ECMO and also the most frequent reason for compelled termination of extracorporeal support it has to be treated in an aggressive 
manner (34). Once the bleeding is under control, systemic anticoagulation is re-started, initially often on a subtherapeutic range.

In patients on VV ECMO we always adapt the pump flow to ensure adequate oxygenation and to accomplish (ultra)protective mechanical ventilation with adequate PEEP levels, low tidal volumes and a driving pressure $(\Delta \mathrm{P})$ of less than $10 \mathrm{cmH}_{2} \mathrm{O}$ if feasible (35). However, in patients after non-elective thoracic surgery and consequently elevated bleeding risk as well as risk for redo surgery, we adjusted our standard protocol. Since most patients with intrathoracic bleeding or infections suffer from diffuse perioperative haemorrhage from the pleural tissue or the lung surface, these patients might benefit from a broad-based contact of the visceral to the parietal pleura. Therefore, we particularly increased the PEEP and temporarily the tidal volume in order to open up residual dystelectases aiming at as much as possible expanded lung parenchyma. This pleural compression between the lung and the chest wall supported by a prolonged chest tube therapy might reduce the risk for intrapleural diffuse bleeding.

Generally, in order to take account for the increased bleeding risk in the perioperative setting, indication setting for any invasive procedure such as placement of additional chest tubes, changes of central venous catheters or even transurethral catheterizations should be critically questioned and minimized whenever possible.

\section{Review of the literature}

The intraoperative use of $\mathrm{VV}$ or VA ECMO in patients undergoing elective, complex thoracic surgery involving the main airway, the heart or the aorta has been reported in various case reports and feasibility studies. These patients suffer from an increased morbidity and mortality rate, which might be rather an attribute of the local advanced disease and the patients' comorbidities (36). But there are also indications for non-elective thoracic surgery procedures in patients while on ECMO support, especially during mid- and long-term support with well-known potential complications (13).

Any surgical intervention during ECMO support has an elevated bleeding risk due to systemic anticoagulation and subsequent coagulation disorders (20,37). According to our experience, these complications steadily increase during longer duration of ECMO therapy: activation of the coagulation system, hyperfibrinolysis, lower platelet count, impaired platelet function, the acquisition of von
Willebrand syndrome and deficiency of certain coagulation components including fibrinogen $(5,10,38)$. ECMO patients may suffer from a consecutive inflammatory response syndrome, caused by the underlying disease, the contact of the blood with the extracorporeal surfaces and the therapy itself (39). According to data of the ELSORegistry 2016, bleeding complications at the surgical site occurred in $16.7 \%$ of patients with a survival rate of $42 \%$ (6). Thoracic surgery was associated with uncontrollable bleeding complications in ECMO patients $(12,22)$. Even the insertion of a chest tube can lead to severe thoracic bleeding complications resulting in a four-time increased mortality $(11,40)$. Therefore, any thoracic intervention (e.g., pleural catheter or drainage) as well as thoracic surgical procedures while on ECMO are always associated with an increased risk of further complications and mortality $(10,13)$. A strict indication selection for any thoracic intervention combined with an adjusted perioperative management (ECMO devices with coated circuits, standard anticoagulation protocol) is highly recommended. Approximately $60 \%$ of our patients with thoracic bleeding complications underwent a previous intervention or thoracic surgery, because the clinical situation of the patient necessitated these diagnostic or therapeutic measurements. Nevertheless, in $40 \%$ of our patients with intrathoracic hemorrhage, the bleeding appeared spontaneously without previous intervention $(10,15,16)$.

Even after primarily successful thoracic surgery and stopping of all systemic anticoagulation, almost half of our patients with intrathoracic bleeding needed repeated surgery due to recurrent bleeding (10). Since in most patients there were diffuse intrathoracic bleedings and also postoperatively the bleeding tendency persisted, packing of the thoracic cavity was the only way for surgical bleeding control in many. Based on our experience, the combination of the following perioperative actions might reduce the risk of further bleeding complications:

(I) Accurate intraoperative surgical bleeding control (especially of the pleural surface; e.g., coagulation, use of fibrin glue);

(II) Liberally packing of the pleural cavity in case of persistent diffuse bleeding;

(III) Prolonged chest tube therapy including frequent lavage to avoid clogging;

(IV) Resting of systemic anticoagulation and a purposeful application of coagulation factors;

(V) Application of an adapted weaning procedure. 


\section{Conclusions}

Strict indication for non-elective thoracic surgery in patients under support with ECMO is highly recommended. Both the underlying disease for the need of ECMO and lifethreatening thoracic complications while on ECMO, too, might necessitate urgent invasive thoracic interventions. Based on our data, the mortality rate was increased in patients with thoracic bleeding complications or after non-elective thoracic surgery. Therefore, we strongly recommend a primary conservative approach and every time careful assessment of thoracic interventions, when clinically justifiable. Furthermore, a modified perioperative coagulation management and weaning protocol were suggested in these patients as well as a prolonged chest tube therapy in order to minimize the risk of recurrent bleeding or repeated surgery.

\section{Acknowledgments}

Funding: None.

\section{Footnote}

Provenance and Peer Review: This article was commissioned by the Guest Editors (Andrea Dell'Amore and Nizar Asadi) for the series "Mechanical Extracorporeal CardioRespiratory Supports in General Thoracic Surgery" published in Current Challenges in Thoracic Surgery. The article has undergone external peer review.

Conflicts of Interest: The authors have completed the ICMJE uniform disclosure form (available at https://ccts. amegroups.com/article/view/10.21037/ccts-20-141/coif). The series "Mechanical Extracorporeal Cardio-Respiratory Supports in General Thoracic Surgery" was commissioned by the editorial office without any funding or sponsorship. The authors have no other conflicts of interest to declare.

Ethical Statement: The authors are accountable for all aspects of the work in ensuring that questions related to the accuracy or integrity of any part of the work are appropriately investigated and resolved.

Open Access Statement: This is an Open Access article distributed in accordance with the Creative Commons Attribution-NonCommercial-NoDerivs 4.0 International License (CC BY-NC-ND 4.0), which permits the non- commercial replication and distribution of the article with the strict proviso that no changes or edits are made and the original work is properly cited (including links to both the formal publication through the relevant DOI and the license). See: https://creativecommons.org/licenses/by-nc-nd/4.0/.

\section{References}

1. Roumy A, Liaudet L, Rusca M, et al. Pulmonary complications associated with veno-arterial extra-corporeal membrane oxygenation: a comprehensive review. Crit Care 2020;24:212.

2. Müller T, Bein T, Philipp A, et al. Extracorporeal pulmonary support in severe pulmonary failure in adults: a treatment rediscovered. Dtsch Arztebl Int 2013;110:159-66.

3. Combes A, Hajage D, Capellier G, et al. Extracorporeal membrane oxygenation for severe acute respiratory distress syndrome. N Engl J Med 2018;378:1965-75.

4. Extracorporeal Life Support Organization. ECLS Registry Report International Summary. 2019. Accessed 13 May 2020. Available online: https://www.elso.org/Portals/0/ Files/Reports/2019/International\%20Summary $\% 20$ January\%202019\%20page\%201.pdf

5. Oliver WC. Anticoagulation and coagulation management for ECMO. Semin Cardiothorac Vasc Anesth 2009;13:154-75.

6. Thiagarajan RR, Barbaro RP, Rycus PT, et al. Extracorporeal Life Support Organization Registry International Report 2016. ASAIO J 2017;63:60-7.

7. Aubron C, DePuydt J, Belon F, et al. Predictive factors of bleeding events in adults undergoing extracorporeal membrane oxygenation. Ann Intensive Care 2016;6:97

8. Kreyer S, Muders T, Theuerkauf N, et al. Hemorrhage under veno-venous extracorporeal membrane oxygenation in acute respiratory distress syndrome patients: a retrospective data analysis. J Thorac Dis 2017;9:5017-29.

9. Enger T, Philipp A, Videm V, et al. Prediction of mortality in adult patients with severe acute lung failure receiving veno-venous extracorporeal membrane oxygenation: a prospective observational study. Crit Care 2014;18:R67.

10. Ried M, Sommerauer L, Lubnow M, et al. Thoracic Bleeding Complications in Patients with Venovenous Extracorporeal Membrane Oxygenation. Ann Thorac Surg 2018;106:1668-74.

11. Joshi V, Harvey C, Nakas A, et al. The need for thoracic surgery in adult patients receiving extracorporeal membrane oxygenation: a 16-year experience. Perfusion 
2013;28:328-32.

12. Taghavi S, Jayarajan SN, Mangi AA, et al. Examining Noncardiac Surgical Procedures in Patients on Extracorporeal Membrane Oxygenation. ASAIO J 2015;61:520-5.

13. Sommerauer L, Philipp A, Lubnow M, et al. NonElective Thoracic Surgery in Patients with Respiratory Insufficiency During Support with Veno-Venous Extracorporeal Membrane Oxygenation. Zentralbl Chir 2019;144:93-9.

14. Schmid C, Philipp A, Mueller T, et al. Extracorporeal life support - systems, indications, and limitations. Thorac Cardiovasc Surg 2009;57:449-54.

15. Malfertheiner MV, Pimenta LP, Bahr VV, et al. Acquired von Willebrand syndrome in respiratory extracorporeal life support: a systematic review of the literature. Crit Care Resusc 2017;19:45-52.

16. Müller T, Philipp A, Luchner A, et al. A new miniaturized system for extracorporeal membrane oxygenation in adult respiratory failure. Crit Care 2009; 13:R205.

17. Schmid C, Philipp A, Hilker M, et al. Venovenous extracorporeal membrane oxygenation for acute lung failure in adults. J Heart Lung Transplant 2012;31:9-15.

18. Ried M, Bein T, Philipp A, et al. Extracorporeal lung support in trauma patients with severe chest injury and acute lung failure: a 10-year institutional experience. Crit Care 2013;17:R110.

19. Zangrillo A, Landoni G, Biondi-Zoccai G, et al. A metaanalysis of complications and mortality of extracorporeal membrane oxygenations. Crit Care Resusc 2013;15:172-8.

20. Aubron C, Cheng AC, Pilcher D, et al. Factors associated with outcomes of patients on extracorporeal membrane oxygenation support: a 5-year cohort study. Crit Care 2013;17:R73.

21. Mazzeffi M, Greenwood J, Tanaka K, et al. Bleeding, Transfusion, and Mortality on Extracorporeal Life Support: ECLS Working Group on Thrombosis and Hemostasis. Ann Thorac Surg 2016;101:682-9.

22. Marasco SF, Preovolos A, Lim K, et al. Thoracotomy in adults while on ECMO is associated with uncontrollable bleeding. Perfusion 2007;22:23-6.

23. Fierro MA, Daneshmand MA, Bartz RR. Perioperative Management of the Adult Patient on Venovenous Extracorporeal Membrane Oxygenation Requiring Noncardiac Surgery. Anesthesiology 2018;128:181-201.

24. McRae K, de Perrot M. Principles and indications of extracorporeal life support in general thoracic surgery. J Thorac Dis 2018;10:S931-46.
25. Peris A, Lazzeri C, Cianchi G, et al. Clinical significance of echocardiography in patients supported by venousvenous extracorporeal membrane oxygenation. J Artif Organs 2015;18:99-105.

26. Douflé G, Roscoe A, Billia F, Fan E. Echocardiography for adult patients supported with extracorporal membrane oxygenation. Crit Care 2015;19:326.

27. Heilmann C, Geisen U, Beyersdorf F, et al. Acquired von Willebrand syndrome in patients with extracorporeal life support (ECLS). Intensive Care Med 2012;38:62-8.

28. Odonkor PN, Stansbury L, Garcia JP, et al. Perioperative Management of Adult Surgical Patients on Extracorporeal Membrane Oxygenation Support. J Cardiothorac Vasc Anesth 2013;27:329-44.

29. Schmidt M, Stewart C, Bailey M, et al. Mechanical ventilation management during extracorporeal membrane oxygenation for acute respiratory distress syndrome: a retrospective international multicenter study. Crit Care Med 2015;43:654-64.

30. Terragni PP, Del Sorbo L, Mascia L, et al. Tidal volume lower than $6 \mathrm{ml} / \mathrm{kg}$ enhances lung protection: Role of extracorporeal carbon dioxide removal. Anesthesiology 2009;111:826-35.

31. Bartlett RH. Physiology of gas exchange during ECMO for respiratory failure. J Intensive Care Med 2017;32:243-8.

32. Sklar MC, Sy E, Lequier L, et al. Anticoagulation Practices during Venovenous Extracorporeal Membrane Oxygenation for Respiratory Failure. A Systematic Review. Ann Am Thorac Soc 2016;13:2242-50.

33. Anselmi A, Guinet P, Ruggieri VG, et al. Safety of recombinant factor VIIa in patients under extracorporeal membrane oxygenation. Eur J Cardiothorac Surg 2016;49:78-84.

34. Lotz C, Streiber N, Roewer N, et al. Therapeutic Interventions and Risk Factors of Bleeding During Extracorporeal Membrane Oxygenation. ASAIO J 2017;63:624-30.

35. Serpa Neto A, Schmidt M, Azevedo LC, et al. ReVA Research Network and the PROVE Network Investigators. Associations between ventilator settings during extracorporeal membrane oxygenation for refractory hypoxemia and outcome in patients with acute respiratory distress syndrome: a pooled individual patient data analysis: Mechanical ventilation during ECMO. Intensive Care Med 2016;42:1672-84.

36. Koryllos A, Lopez-Pastorini A, Galetin T, et al. Use of Extracorporeal Membrane Oxygenation for Major 
Cardiopulmonary Resections. Thorac Cardiovasc Surg 2020. [Epub ahead of print]. doi:10.1055/s-0040-1708486.

37. Panigada M, Artoni A, Passamonti SM, et al. Hemostasis changes during veno-venous extracorporeal membrane oxygenation for respiratory support in adults. Minerva Anestesiol 2016;82:170-9.

38. Tauber H, Ott H, Streif W, et al. Extracorporeal membrane oxygenation induces short-term loss of highmolecular-weight von Willebrand factor multimers.

doi: $10.21037 /$ ccts-20-141

Cite this article as: Ried M, Sommerauer L, Markowiak T, Wiesner S, Mueller T, Philipp A, Unterbuchner C, Creutzenberg M, Lunz D, Hofmann HS. Non-elective thoracic surgery in patients supported by veno-venous (VV) or venoarterial (VA) extracorporeal membrane oxygenation (ECMO). Curr Chall Thorac Surg 2020.
Anesth Analg 2015;120:730-6.

39. Esper SA, Levy JH, Waters JH, et al. Extracorporeal membrane oxygenation in the adult: a review of anticoagulation monitoring and transfusion. Anesth Analg 2014;118:731-43.

40. Repessé X, Au SM, Bréchot $\mathrm{N}$ et al. Recombinant factor VII a for uncontrollable bleeding in patients with extracorporeal membrane oxygenation: report on 15 cases and literature review. Crit Care 2013;17:R55. 